livraisons

d'Histoire

de l'Architecture

\section{Livraisons de l'histoire de l'architecture}

$17 \mid 2009$

Émotions patrimoniales I

\title{
Saint Sernin de Toulouse : la basilique de la discorde
}

Saint Sernin of Toulouse : the basilica of dissension

Saint Sernin von Toulouse : die Basilika der Zwietracht?

\section{Bérénice Waty}

\section{(2) OpenEdition}

Journals

Édition électronique

URL : http://journals.openedition.org/lha/210

DOI : 10.4000//ha. 210

ISSN : 1960-5994

Éditeur

Association Livraisons d'histoire de l'architecture - LHA

Édition imprimée

Date de publication : 10 juin 2009

Pagination : 73-91

ISSN : 1627-4970

Référence électronique

Bérénice Waty, "Saint Sernin de Toulouse : la basilique de la discorde ", Livraisons de l'histoire de l'architecture [En ligne], 17 | 2009, mis en ligne le 10 juin 2011, consulté le 21 mars 2020. URL : http:// journals.openedition.org/lha/210; DOI : https://doi.org/10.4000/lha.210

Ce document a été généré automatiquement le 21 mars 2020.

Tous droits réservés à l'Association LHA 


\section{Saint Sernin de Toulouse : la basilique de la discorde}

Saint Sernin of Toulouse : the basilica of dissension

Saint Sernin von Toulouse : die Basilika der Zwietracht?

\section{Bérénice Waty}

1 «Celui qui fut une sorte de Léonard-Jules Vinci-Verne [...] qui avait le mieux et continûment célébré les noces renouvelées du passé et du présent » était honoré en 1980 par une exposition au Grand Palais ; Viollet-le-Duc n'était plus «bon ou méchant : il existait ", selon les mots de Bruno Foucart, commissaire de l'événement, qui se félicitait du « véritable revival » en l'honneur de ce "premier des architectes-savantspoètes du XIX ${ }^{e}$ siècle $^{1}$ ». Évacués donc les temps où l'ami de Prosper Mérimée était conspué, où son travail à Notre-Dame de Paris ou au château de Pierrefonds n'attirait que foudres et polémiques. Viollet-le-Duc, l'«un des plus grands criminels de l'Histoire » qui «a fait disparaître l'âme du passé, crime historique le plus odieux qui puisse être " ${ }^{2}$, se voyait réhabilité à la fin des années 1970, et plus encore, louangé au sein d'expositions, de colloques ou d'ouvrages, tant scientifiques que destinés au grand public.

2 Et pourtant! L'année même du centenaire de sa mort, en 1979, la commission supérieure des monuments historiques (CSMH) clouait au pilori l'intervention de Viollet-le-Duc sur la basilique Saint-Sernin de Toulouse, en retenant le projet de dérestauration présenté par l'architecte en chef des Monuments historiques, Yves Boiret, en charge de l'édifice. "Hier calomnié [...] presque trop célébré3 [en 1980]», l'architecte à la légende noire se trouvait une nouvelle fois attaqué pour son entreprise dans la cité palladienne, en raison notamment du choix peu judicieux fait par lui d'un matériau qui s'était révélé trop fragile avec les années. Ironie de l'histoire, de ses relectures et ré-écritures, c'est déjà pour ce même monument de la Ville rose que le grand architecte parisien avait dû affronter une controverse féroce menée par les élites locales alors qu'il tentait de parer aux outrages du temps. Dérision redoublée par l'observation des acteurs de ces débats d'alors et de leurs arguments: la société archéologique du midi de la France (SAMF) n'avait pas eu de mots assez durs contre 
l'expert du XIX siècle, opposant à sa restauration le " concert unanime de blâmes et de regrets $»^{4}$ qui l'avait accueillie. En 1989 , le même organe qui vilipendait un siècle plus tôt Viollet-le-Duc encense son ouvrage et défend son héritage contre un architecte jugé trop parisien, que l'on renvoie aux incongruités de son projet. Mais on verra, par la suite, que cette répétition de l'histoire offre d'autres manifestations surprenantes.

Ainsi, à un siècle d'écart, au sujet du même monument, deux hommes vont-ils voir leur entreprise de restauration cruellement dénigrée, être attaqués pour leurs compétences mêmes et devoir affronter la capitale du Languedoc.

Sans doute la nature même du lien que les Toulousains entretiennent avec cet édifice at-elle un rôle éminent dans ce retour des polémiques. "Saint-Sernin n'est pas une église de Toulouse, c'est Toulouse même dans sa personnification la plus complète et la plus éloquente [...]. L'histoire de Saint-Sernin se trouve liée à toutes les grandeurs, à toutes les légendes poétiques et guerrières $\mathrm{du}^{\mathrm{Midi}}{ }^{5} »$ : ce constat nous accompagnera tout au long de notre réflexion sur les querelles que cette basilique n'a pas cessées de déclencher entre les habitants de la ville et le pouvoir qui en a la charge depuis qu'elle a été classée monument historique en 1838. Ouvrage métonymique, qui explique l'âpreté avec laquelle les Toulousains se sont toujours opposés à des restaurations pensées et effectuées par la capitale. Leur lutte, à chaque fois combative et argumentée, est rapportée à la nature légendaire de la population : «Ce peuple formé par le mélange heureux des Celtes et des Romains, unissant en lui le tempérament hardi, querelleur et belliqueux de son ancêtre, le Volce Tectosage, à l'esprit avisé, entreprenant et politique du Latin ${ }^{6}$. » Un héritage qui pourrait justifier tant au XIX ${ }^{e}$ qu'au $\mathrm{XX}^{\mathrm{e}}$ siècle la nature batailleuse des adversaires autochtones des deux fonctionnaires de l'État.

Si l'effet miroir joue pleinement dans ces deux campagnes de restauration, la controverse suscitée par le projet Boiret fait apparaître une nouveauté en matière de patrimoine. En effet, si les acteurs qui s'affrontent sur le sort architectural de la basilique sont les mêmes dans les deux cas, si la nature du conflit repose sur des conceptions et théories antagonistes de l'art de restaurer, les années 1980 voient émerger deux nouveaux intervenants: la société civile, avec ses associations, et les médias qui relaient, et parfois arbitrent, les passes d'armes entre les camps opposés.

6 En 2000, dans le cadre d'un rapport sur cette " affaire ${ }^{7}$ » qui nous avait été commandé par la direction régionale des affaires culturelles de Midi-Pyrénées (DRAC), nous avions rencontré les protagonistes du conflit (élus, journalistes, associatifs), donnant lieu à une dizaine d'entretiens dont nous avions étayé l'analyse par une revue de presse locale et nationale. Nous nous proposons donc de revenir sur la " guerre des mirandes ${ }^{8}$ » afin d'en expliciter les rouages. Il nous avait semblé pouvoir les saisir dans une lecture ethnocentrée du projet de Boiret produite par la cité palladienne. Tentons de restituer l'ambiance d'alors, par l'évocation des différents actes de cette dramaturgie et un retour sur quelques éléments propres à la société civile toulousaine

\section{Bataille autour d'une restauration : le cas des années 1980}

7 « Avec Viollet-le-Duc la qualité des analyses, la maîtrise des chantiers sont telles que la polémique n'a plus de sens qu'à la hauteur des principes $^{1} »$ : ce constat éclaire l'opposition aux travaux envisagés par Boiret. Les acteurs en présence, pour partie, 
fixeront leur discorde autour de théories architecturales et de lectures antagonistes de l'essence même d'une restauration. Pour saisir la nature de cette controverse, il faut en consigner quelques temps forts.

\section{Scientifiques et professionnels opposés : Durliat et Boiret polémiquent}

Depuis les années 1960, la basilique connaît différentes campagnes de travaux. Boiret se lance à son tour dans le rétablissement des parties hautes et extérieures, fortement détériorées. Il a fondé les arguments de son projet sur toute l'iconographie connue du monument et s'inspire des notes et travaux de Viollet-le-Duc lors de sa propre intervention sur ce haut lieu de l'art roman en France. Mais, dans les sphères universitaires et patrimoniales, le professeur Marcel Durliat soulève le problème de la « dé-violletisation » qui se profile. Dès lors, commence une passe d'armes entre les deux hommes, qui vont faire valoir leurs arguments dans diverses tribunes scientifiques. La querelle est donc connue et chacun des camps s'oppose aux vues et méthodes de l'autre, personne ne désarme.

9 Le nœud de leur désaccord repose sur l'existence ou non d'un " état d'origine » du monument. Les termes employés par Durliat (1980) sont radicaux: il parle de l'« illusion d'un possible retour à l'état d'origine, un tel état n'a jamais existé ». Mais plus encore, il estime qu'il ne fait pas de doute que la volonté de Boiret et de l'administration centrale est de réduire « les apports de l'architecte du XIX siècle ». Il s'agit même selon lui, de supprimer les traces de Viollet-le-Duc: "On passe par un jugement de valeur sur le XIX ${ }^{\mathrm{e}}$ siècle, et plus particulièrement sur l'œuvre de Viollet-leDuc restaurateur ». Il renchérit : "Pour certains de nos contemporains, le cours de la grande histoire, celle qui confère à un édifice un prestige reconnu, en l'enveloppant de cette aura mystérieuse qui fait de lui un "monument historique", s'arrêterait précisément au XIX ${ }^{\mathrm{e}}$ siècle. [...] Des restaurations comme celles de Viollet-le-Duc à Saint-Sernin [...] sont dénoncées comme des initiatives personnelles, hasardeuses, dépourvues d'authenticité, qu'on supporte mais dont on saluerait la disparition avec soulagement [...]. Ses grandes manifestations, comme le Saint-Sernin de Viollet-le-Duc, méritent donc d'être pérennisées en raison de leur valeur de témoignage. »

10 À ces critiques, Boiret rétorque point par point ${ }^{2}$. Il fait état de sa réflexion, menée à partir de documents d'époque, en pointant un "état précédent, parfaitement connu » et « antérieur à 1860 » (1980): il se base sur des calotypes représentant la basilique. Il conteste l'effacement de «l'intervention du XIX siècle ». En étudiant les archives des restaurations de Saint-Sernin, il a acquis la conviction que le projet de Viollet-le-Duc était intéressant, mais que l'architecte n'a pu atteindre ses objectifs dans sa mise en œuvre. De plus, il rappelle le caractère urgent des travaux à cause du choix malheureux d'un matériau inapproprié au XIX ${ }^{e}$ siècle. Ce sera d'ailleurs le principal argument retenu par la commission supérieure des monuments historiques lors du premier vote en janvier 1979: la reconstruction des parties hautes s'imposait, car la pierre et le système d'écoulement des eaux adoptés par son illustre prédécesseur en rendait la restauration impossible.

11 Boiret tente l'apaisement en incitant à «ne pas passionner le débat! ». Il dénonce le risque qui pèse sur la querelle scientifique : «Le débat ne doit en aucun cas être classé sous la rubrique "Affaire de Toulouse" ». L'exhortation lui est inspirée par la situation 
rencontrée au siècle précédent: le destin du monument échappant aux sociétés savantes locales, leurs critiques se firent virulentes à l'encontre de Viollet-le-Duc. L'architecte aurait dénaturé de façon si criante les lignes, les volumes, parfois même les matériaux d'origine, que le congrès de la SAMF de 1874 dresse contre lui un véritable réquisitoire. L'abbé Carrière, qui le présidait, flétrit « les innovations fantaisistes aussi désastreuses pour le monument que fâcheuses pour celui qui les a conçues ${ }^{3}$ ». L'un des symptômes de la controverse apparaissait en filigrane : la crise reflètera les "passions identitaires» de la Ville rose, avant de symboliser une polémique architecturale et théorique.

\section{Un débat public qui transcende les sphères scientifiques}

12 Pendant dix ans, les deux hommes se livrent à une dispute nourrie. Mais comment sortir du statu quo à partir de positions indépassables? Comment la campagne de restauration peut-elle débuter?

13 En 1989, ce débat doctrinal déborde le cadre universitaire et celui des experts pour aboutir sur l'agenda politique et agiter la population toulousaine.

Bien que toujours actif, Durliat est malade; plusieurs de ses anciens élèves prennent sa relève et le 31 mai adressent un courrier au ministère ${ }^{4}$. Ils demandent que le projet Boiret soit rejugé par la commission supérieure des monuments historiques afin de prendre en compte les différents problèmes soulevés lors des échanges des années 1980. Ceci au nom du respect de la basilique et de Viollet-le-Duc. Refus de la Rue de Valois. Mais le groupe ignore cette fin de non-recevoir: une seconde missive, plus argumentative, apporte des éléments inédits au dossier. Les nouvelles recherches d'Odile Foucaud ${ }^{5}$, qui venait d'entrer en possession d'un document jusqu'alors inconnu, prouvaient «que Viollet-le-Duc [avait] respecté, en 1862, les dispositions des toitures romanes redécouvertes lors de la destruction des combles par l'architecte Esquié ». Boiret, contrairement à ses déclarations, ne retournait donc pas à un "état antérieur ". Un nouvel avis de la commission supérieure des monuments historiques est sollicité. La création d'un comité international de parrainage (CIP) est également annoncée. Le 17 novembre 1989, la réponse leur parvient : le dossier sera ré-examiné par la commission supérieure des monuments historiques. Revirement surprenant de la part de l'administration centrale, qui demande à la commission supérieure des monuments historiques de se déjuger et suspend le démarrage des travaux.

Loin de ce lobbying, une exposition ouvre ses portes au musée Saint-Raymond à Toulouse. Intitulée Saint-Sernin de Toulouse, trésors et métamorphoses: deux siècles de restauration, 1802-1989, elle est placée sous la responsabilité de la mairie et de la DRAC. Elle dure du 15 septembre 1989 au 14 janvier 1990. Des maquettes présentent le nouvel aspect du monument envisagé par Boiret: les gens peuvent juger sur pièces les modifications introduites, à partir des calotypes montrant l'état antérieur de la basilique. La manifestation est doublée d'une série de quatre conférences d'historiens et d'architectes.

16 Un élément crucial permet alors d'éclairer le tour que prennent bientôt les événements : l'apparition des médias dans le débat. Elle va accélérer et cristalliser la lutte. Grâce au relais journalistique, différents protagonistes mêlent leur voix à la discussion et prennent position pour ou contre le projet tant sur la place publique toulousaine, qu'au niveau national et international. En effet, le camp de Durliat va 
rapidement trouver la presse régionale pour rendre publiques ses craintes et son opposition au projet. Le ministère tente une ouverture avec une interview accordée par le directeur de la DRAC pour calmer les ardeurs de ses détracteurs ${ }^{6}$. Boiret tente, lui, de communiquer sa position de manière pédagogique et argumentée, à travers deux conférences publiques. Mais dorénavant, c'est la population toulousaine qui entre en lice.

Les défenseurs de Viollet-le-Duc saisissent le premier magistrat de la ville : au mois d'octobre, la municipalité, maire en tête, rentre dans la polémique ${ }^{7}$. Prenant le relais des scientifiques, la presse toulousaine relate leurs divergences depuis 1980. À la pointe de cette médiatisation, La Dépêche du Midi avec son journaliste Philippe Emery ${ }^{8}$. Ce dernier est radical dans son rejet de la proposition de Boiret. Son vocabulaire de polémiste emprunte au registre de la guerre : il accuse l'architecte de "guillotiner", " combattre ", « raser » ou " détruire à néant " pour un "prétendu retour à un état antérieur à 1860 ! »; il faut selon lui « éviter des erreurs graves ».

Le mois de novembre 1989 est sans doute l'un des plus importants des neuf mois que dure cette affaire. Le 15 novembre, la presse diffuse le "manifeste des 36 opposants " qui constitue l'acte de naissance du CIP, réunissant des savants et universitaires du monde entier. Le 23, Boiret donne sa conférence au musée Saint-Raymond : il a fallu « rajouter des chaises » et programmer pour le lendemain même un bis repetita. L'abbé Rocacher, décrivant cette rencontre, fait état d'une tension forte : «La situation était extrêmement orageuse. Il fait une conférence avec une mine de déterré, comme si on allait l'égorger!». La Dépêche du Midi rapporte les réactions de la salle: «Le toit de hangar que vous voulez mettre sur Saint-Sernin, c'est une honte ${ }^{9}$ »"

$\mathrm{Au}$ même moment, les mouvements associatifs toulousains entrent dans l'arène: associations de riverains, de commerçants ${ }^{10}$; les sociétés savantes également : la SAMF et l'association des Toulousains de Toulouse, respectivement le 21 et le 23 novembre, font connaître leurs objections. L'association La Flamme cathare prend aussi position : "La décision et le choix sont le fait d'un aréopage extérieur et parisien. [...]. La basilique n'est pas un simple monument historique. Elle est vivante. Elle est Toulouse. Elle est partie intime de la vie, de l'histoire, de l'âme, de la culture de Toulouse, ainsi que de la civilisation que Toulouse a véhiculée au fil des siècles ${ }^{11}$ ».

Le 27 novembre, intervient un épisode important: la commission supérieure des monuments historiques se réunit une nouvelle fois pour statuer sur le projet Boiret. Les Toulousains sont invités à venir plaider leur cause, des membres du $\mathrm{CIP}^{12}$ font le déplacement. La Commission les auditionne plusieurs heures durant. Le vote final fait état de 19 voix sur 24 en faveur du projet. La commission supérieure des monuments historiques n'a pris en compte ni l'émoi populaire ni les nouveaux arguments archéologiques et architecturaux. Pour Durliat, il ne fallait rien en attendre: "J'ai considéré que cette commission n'avait pas changé depuis que j'en étais membre en 1979 [...]. Son vote massif, quasi unanime, montre qu'elle n'est pas crédible [...], le résultat est suspect. [...] sans tenir compte de l'opinion locale ${ }^{13}$.» Les propos de Bernard Durand $^{14}$, alors président de l'Association pour la sauvegarde du patrimoine, la protection de l'environnement et l'amélioration du cadre de vie des Toulousains (ASPECT), corroborent cette impression: «On a reçu ça comme un refus parisien de vouloir nous écouter ; c'était comme une baffe [...] ; on a continué à lutter.» De son côté, le CIP estimait avoir accompli sa mission: les scientifiques voulaient faire un «baroud d'honneur », comme Rocacher s'en explique : «On n'y croyait pas trop, non. 
On disait que cette affaire-là était un petit peu dure à avaler. Nous avions des arguments solides pour argumenter; mais nous avions tous assez de connaissances du poids de l'administration pour ne pas nous faire trop d'illusions [...]. On partait en sachant que ça serait le pot de terre contre le pot de fer. » En obtenant un nouvel avis de la commission supérieure des monuments historiques, ils avaient remporté une sorte de victoire morale qui avait valeur symbolique.

Ensuite, comme l'affirme Rocacher, «les choses ont dépassé » le CIP et les actions émanant de mouvements militants autonomes ont pris le dessus.

Une autre phase de la controverse apparaît en effet, avec à sa tête l'association ASPECT. Celle-ci invite la population à envoyer des cartes postales du monument au président de la République afin de lui signifier sa détermination à conserver Saint-Sernin en l'état. Leurs slogans sont sans équivoque: "Telle qu'elle est, elle me plaît!» ou « Touche pas à mon Saint-Sernin! ». Elle lance également une pétition, qui recevra près de 13000 signatures en deux semaines ${ }^{15}$. Mais le point d'orgue de la contestation est sans nul doute « l'occupation » de la basilique par l'association.

Le 14 juin 1990, ASPECT décide de mener une « opération coup de poing » afin de faire «son possible pour la basilique», selon Durand. Un camping-car, des tentes apparaissent devant la Porte du Mège. Les membres de l'association s'y installent en improvisant des stands sur des tréteaux pour montrer à la population dessins, photographies, et revenir longuement sur la thèse d'Odile Foucaud. Des banderoles sont posées sur les grilles de la basilique, des pancartes jonchent la place. La Dépêche du Midi parle « d'occupation » avec « des tentes dans les jardins » et affirme que l'association va «faire la fête au ministre $»^{16}$. La basilique sera ainsi « habitée » du 14 au 30 juin 1990. Les protestataires reçoivent la visite de membres du CIP dont le soutien et la caution scientifique tendaient à attester la vérité des informations alors diffusées. Que la police municipale ne fasse pas évacuer les lieux semble ajouter à cette légitimation de leur action. Durand parle d'un véritable succès populaire: «Les gens passaient, nous parlaient et signaient la pétition. »

Cette mise en scène leur vaudra les invectives du directeur régional des affaires culturelles : " Nous ne sommes pas des hooligans! "s'insurgera Durand à son endroit. Les déclarations de l'époque sont énergiques : "Notre intention est de résister pied à pied à la reprise des travaux", "Nous sommes prêts à nous opposer par tous les moyens dès que les travaux commenceront [...] avec des actions spectaculaires, comme on s'oppose à l'implantation d'une centrale nucléaire!». En se comparant aux écologistes et en mettant en parallèle leur basilique dé-violletisée avec une centrale nucléaire, les militants du patrimoine affichent une détermination confinant parfois au jusqu'au-boutisme, voire au fanatisme. 


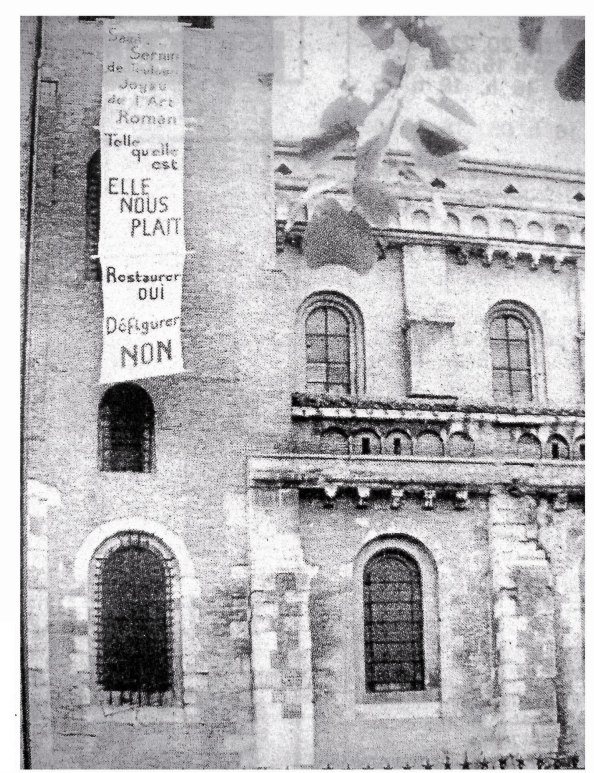

L'auteur remercie le service de la documentation du journal qui lui a accordé le droit de reproduire cette image. Photographie accompagnant l'article de Philippe Emery, « Les anti-Boiret campent sur leurs positions », La Dépêche du Midi, 15/06/1990

(C) La Dépêche du Midi

Jugeant que le procédé est "un abus de confiance », le comité pour la transparence (CPT) va, lui aussi, aller défendre "son" propre Saint-Sernin. Il improvise sur des pancartes un récapitulatif de l'histoire des restaurations de la basilique et une présentation des travaux envisagés par Boiret. Bravant le monopole d'ASPECT, ses membres s'installent à quelques mètres de l'autre campement. Guy de Toulza ${ }^{17}$, créateur du CPT, n'admet pas cette « désinformation totale». Il déclare ainsi dans Le Journal de Toulouse: "Notre démarche se situe dans un but honnête, sans ambition politique ou autre [...]. Nous renseignons sur ce qui a été occulté ${ }^{18}$.» De Toulza donnera une conférence improvisée sur le parvis du monument devant trois cents personnes.

En prétendant agir en historiens, les membres du CPT stigmatisaient le mode d'action d'ASPECT et s'en démarquaient profondément : le CPT ne « squattait » pas, il se trouvait " devant la basilique », pour ce qui n'était pas une " occupation ».

À travers ces deux mouvements, transparaît le rôle prépondérant de la presse locale : elle prend une part décisive à la création de l'affaire, rythme son existence durant un an et contribue à entretenir la tension. Le registre lexical auquel les journaux ont recours laisse peu de place à une présentation objective de la restauration: " polémique ", relevée à 37 reprises, est l'expression la plus utilisée. Suivent « bataille " (25), «querelle » (19), " affaire » et «contestations » (12), "subir un siège » (9) et " combat » (8). Le mois de novembre 1989 connait la plus forte production d'articles dans la presse locale (26 articles sur un total de dix mois et de 150 articles), jamais égalée par la suite. Un vocabulaire religieux surgit aussi - «Priez pour Saint-Sernin », 
« Désacraliser »--, comme si seule la religion pouvait sauver ce monument-phare sur la route des pèlerins ralliant Saint-Jacques de Compostelle.

La Dépêche du Midi et Le Journal de Toulouse sont les deux titres les plus actifs, avec un total de 74 articles pour le premier et 35 pour le second. Ils divergent rapidement dans leur façon de relater l'affaire. La Dépêche prend parti contre le projet Boiret ${ }^{19}$, Le Journal de Toulouse affichant plus de neutralité. Mais il sera l'unique tribune du CPT, et l'on est tenté d'y déceler une prise de position en faveur de la dérestauration.

On note dans leurs colonnes la convocation d'un « reste cathare ». L'enrôlement dans la lutte de l'association La Flamme Cathare pourrait relever de l'anecdote : néanmoins il atteste la dimension régionaliste très forte de ce conflit et le marque de son sceau dans une vision anti-jacobine et anti-parisienne. D'ailleurs Emery, de La Dépêche du Midi, s'en inspire lui-même: "Le vieux fond cathare des Toulousains, toujours prompts à se révolter contre les directives venues de Paris, vient un peu plus passionner le débat ${ }^{20}$. »

C'est logiquement que l'affrontement Paris-Toulouse, centre-périphérie, apparaît alors dans leurs pages. Ainsi, Emery stigmatise le « caractère autoritaire et centralisateur de cette décision" (Rue de Valois, commission supérieure des monuments historique, l'architecte parisien) et le centralisme jacobin qui s'imposerait aux responsables de découvertes archéologiques, aux érudits locaux et à la population toulousaine : "On entend ainsi donner des leçons de style aux provinciaux d'Occitanie. » Il est à souligner que les services du Ministère sont absents de la presse.

31 Après presque une année de déclarations acerbes et d'événements divers, les travaux vont néanmoins commencer dans une indifférence totale. Le ministre Jack Lang avait pris sa décision en juin. L'occupation de la basilique levée, le chantier s'ouvre courant juillet 1990. Tous les protagonistes de l'affaire ont déserté, au premier rang desquels le CIP et la mairie. Durand estime que "les pouvoirs publics ont joué la sécurité » et "monté les échafaudages durant les vacances", quand personne ne paraissait en mesure de s'opposer à cette issue.

Polémique étonnante que celle-ci. Dix années de controverse doctrinale et architecturale avec Durliat. Une année de prise de parole par des acteurs multiples. Opposition à des travaux de restauration, rejet des compétences professionnelles d'un représentant de l'État et attaques personnelles. Plasticité des modes de mobilisation pour prendre position: lettres ouvertes dans la presse ou auprès des pouvoirs dirigeants, envois d'une pétition, conférences dans des institutions culturelles ou in situ et impromptues, sit-in et menaces de représailles électorales, lobbying dans les sphères décisionnelles. Profusion d'avis des uns et des autres : des comités créés dans l'urgence du débat, des sociétés savantes qui renient leurs prédécesseurs en encensant Viollet-leDuc, des mouvements populaires qui s'échauffent, des élus locaux qui endossent différents discours selon que leurs interlocuteurs soient toulousains ou parisiens.

Elle révèle un bégaiement de l'histoire, la basilique n'ayant jamais pu être restaurée sans éclats de voix et de colère. De 1815 à 1845, déjà, trois affaires attisent les tensions autour de travaux ${ }^{21}$. De 1860 à 1879, Viollet-le-Duc se heurte à des critiques féroces. Enfin, « la guerre des mirandes » et la vindicte publique.

Mais in fine le ressort de la polémique n'était-il pas de voir surseoir au projet? Le sentiment d'appartenance à une identité toulousaine se voyait remis en cause. Autour du monument en jeu, tous se réunissaient contre un ennemi commun fantasmé, dans une tentative pour l'identifier et le percevoir de nouveau. La société locale est 
«toujours un jeu à trois, jamais à deux : il y a toujours les indigènes, le territoire et l'étranger ${ }^{22}$. " Ici, Boiret et son projet endossent le dernier rôle. " La ville a joué le rôle mémorial (forcément patrimonial) de monument : objet qui possédait [...], le double et merveilleux pouvoir d'enraciner ses habitants dans l'espace et le temps ${ }^{23} "$ : la mémoire et l'identité de la cité palladienne sont alors devenues des protagonistes du conflit.

\section{Micro-crises fratricides dans la vie toulousaine}

35 "Étudiée avec passion, source de querelles et divergences parfois entre les chercheurs ${ }^{1}$ ", cette basilique a attisé les antagonismes au-delà des spécialistes : la mairie et les élus, les pouvoirs religieux, les élites locales ont toujours pris part à sa destinée. En 1989, ils étaient présents, rejoints par les médias, les pouvoirs décentralisés, les associations citoyennes.

Par leurs ventes, La Dépêche du Midi et Le Journal de Toulouse ont cherché à affirmer, l'un face à l'autre, leur hégémonie éditoriale. Lors de la polémique, les oppositions internes à la Ville Rose ne s'arrêtèrent pas là: la virulence et le fondement même de la controverse sont à mettre en relation avec le climat inédit qui règne à Toulouse à cette époque.

\section{Une identité toulousaine à reconfigurer}

37 Le patrimoine de Toulouse va se transformer en ressources à la fin des années 1980 : «Des ressources pour ré-ancrer, ré-enraciner des identités défaites par la vitesse et la mobilité généralisées ${ }^{2} »$. En 1989, la ville a un nouveau visage: sa population a fortement augmenté, du fait principalement des nombreuses embauches dans l'aérospatiale. Elle est un chantier ouvert et permanent. Des projets sont lancés pour satisfaire socialement et culturellement cette nouvelle population : renouvellement du parc immobilier, création du Zénith, ou implantation du métropolitain symbolisent cette politique.

Ces différentes modernisations sont parfois critiquées par certaines associations, comme des comités écologistes ou les Amis du Vieux Toulouse ${ }^{3}$. Par-delà les controverses, l'intégration des nouveaux habitants connaît des difficultés et deux populations cohabitent à Toulouse : l'ancienne et celle qui vient d'arriver. Il s'agit de les ré-unir afin de ré-affirmer une identité solidaire et unique. La querelle autour de la basilique y concourt: le patrimoine va exercer une fonction de médiation entre l'individu et la société, permettant l'affirmation d'un groupe, "les Toulousains ». Ici, «le patrimoine ne concerne pas l'intégration à la société, mais l'intégration à la communauté : il a une fonction pratique ${ }^{4} »$.

Dans cette affaire, différents processus se sont imbriqués. Pour les Toulousains d'adoption récente, il s'agissait de reconnaître l'importance de Saint-Sernin en tant qu'emblème de la ville et de sceller ainsi leur intégration dans le groupe. Pour les anciens, la défense du monument leur permettait de confirmer cet enracinement de toujours. La mobilisation rend possible la communion et la concorde de tous ces différents Toulousains. Il s'est agi d'une quête fusionnelle, en faveur d'une affirmation identitaire. 


\section{Quête d'unité mais luttes intestines}

40 ont prévalu. Aussi, la lutte pour la basilique peut-elle se lire comme une querelle des "Anciens et des Modernes" transposée dans le militantisme patrimonial et comme le témoignage d'attachements différents au monument.

41 Le premier type d'attitude est celui d'une opposition classique avec un seul mode d'action : la lettre ouverte. La SAMF, les Amis du Vieux Toulouse, la Flamme cathare, les Toulousains de Paris, les Amis de Saint-Sernin, ont tous signé une ou plusieurs lettre(s) où ils exprimaient leur désaccord face à une "dé-violletisation». Les élèves du professeur Durliat et le CIP ont œuvré de même. Dans cet exercice de style, seul compte l'avis des personnes signataires. Ces stratégies sont dictées par une vision traditionnelle du pouvoir où seules les associations ayant acquis le rang d'institutions peuvent prétendre s'adresser aux officiels, au nom d'un bien général qu'elles sont censées incarner.

Cons les années 1980, la population s'en mêle. C'est là qu'entrent en scène ASPECT et le Comité de quartier Arnaud Bernard, dans une moindre mesure : ils se saisissent de ce besoin populaire de revendiquer une appartenance commune à un lieu. Loin de les ignorer, ils enjoignent au contraire les Toulousains à les rejoindre et à se manifester, via les pétitions qui sont une prise directe de citoyenneté, via «l'occupation». Si ces manifestations visaient à obtenir l'attention des médias, elles cherchaient aussi à rassembler la population toulousaine. Un second mode d'action les distingue ici de ceux qui recourent aux procédés connus (lettres et lobbying), ils innovent dans leur opposition au projet officiel. ASPECT développe une contestation bruyante et avantgardiste pour se faire entendre. Ses actions sont empreintes de l'activisme des années 1970 : sit-in, concert, "occupation", escalade sur le clocher, volonté d'être au plus proche des gens pour dialoguer. Elle occupe le terrain, est physiquement au chevet de la basilique. Peu importe le succès ou non de ses manifestations, leurs existences seules les font se détacher du lot des autres associations. Durand revendique cette différence : «Nous avions une démarche évolutive, c'est-à-dire que nous nous démarquions très clairement de ce qui existait à l'époque [...] de ce que nous appelons des associations d"'ayatollahs", fixées sur le passé et pour qui rien ne doit bouger. "

Mais les oppositions entre les associations tiennent aussi à la visée du combat mené. Pour le CIP, il fallait avant tout se faire entendre, protester pour la forme car ils se sentaient tous «moralement » contraints de manifester leur désaccord. Pour ASPECT il s'agissait de défendre Viollet-le-Duc: "C'était comme si on voulait réécrire Chateaubriand, pour lui donner le style de Marguerite Duras!» "Cette œuvre était intouchable!», tel était son leitmotiv. D'autre part apparaît un autre registre de la mobilisation: c'est une lutte "NIMBY ${ }^{5}$ ». Le comité de quartier Arnaud Bernard, les riverains de la basilique ou les commerçants non sédentaires de la place Saint-Sernin, ne voulaient pas que la physionomie de leur monument soit modifiée car leur environnement en aurait été transformé. Il s'agissait avant tout d'une contestation riveraine et individualiste. Enfin le tissu associatif était marqué par la concurrence. Plusieurs associations signent une lettre commune, critiquant le monopole médiatique d'ASPECT et lui reprochant de mener ses actions sans concertation aucune. 


\section{Conclusion}

Avec l'affaire de Saint-Sernin de Toulouse, Boiret, propulsé au cœur d'une polémique scientifique, puis médiatique, connaît la même situation que Viollet-le-Duc dans les années 1840. Dans un article en 1989, il se compare, à bon escient, à son illustre prédécesseur. Il conclut qu'après avoir été critiqué en son temps, Viollet-le-Duc avait été reconnu et défendu par les contemporains: il en espérait de même pour lui. L'histoire semble lui donner raison: au milieu des années 1990, il se voit remettre des mains du maire une distinction honorifique pour l'ensemble de son travail à Toulouse, devant un parterre d'invités, ceux-là mêmes qui menaient la fronde. Autre pied de nez : les cartes postales touristiques actuelles reproduisent une vue des mirandes dans le nouvel aspect que leur a donné Boiret, et leur légende indique que c'est à Viollet-le-Duc qu'on doit leur restauration. Le pari serait peut-être gagné ?

«Toute intervention est fille de son temps, les humeurs et les goûts l'emportent toujours ${ }^{1} »$ : tel pourrait être le sous-titre de la polémique ici décrite, tant réécritures et relectures polymorphes des doctrines architecturales et du militantisme se sont données à voir. Mais tant aussi le caractère batailleur et les particularités de la Ville rose ont fait de cette péripétie une illustration de sa coutumière opposition à Paris et à l'État.

Ce cas d'« émotion patrimoniale » est aussi emblématique des années 1980 : face à une administration " autiste ${ }^{2}$ », la population a voulu " exercer un droit de regard ${ }^{3}$ » sur son action dans un mouvement de démocratisation du patrimoine. En matière de monument, l'objet de la dispute, quel qu'il soit, s'efface derrière l'événement lui-même qu'est la polémique : ainsi l'avènement de "nouvelles passions patrimoniales ${ }^{4}$ ", et d'«identités fusionnelles limitées au temps bref d'une communauté d'action dans un pur concentré d'énergie collective ${ }^{5} »$ est-il perceptible.

\section{ANNEXES}

\section{REVUE DE PRESSE}

- 14/09/1989 : « Saint-Sernin revisitée ». La Dépêche du Midi / Joël Saurin.

- 16/09/1989 : « Saint-Sernin d'hier et d'aujourd'hui ». La Dépêche du Midi / Joël Saurin.

- 16-17/09/1989 : «Une restauration obligée ». Le Journal de Toulouse / Pierre Rey.

- 16-17/09/1989 : « La dynamique forte et incitative (interview de Patrice Béghain ${ }^{6}$ )».

- Septembre 1989 : « Saint-Sernin, 1860-1862 : 28 feuillets de dessins inédits de Viollet-

le-Duc et Esquié ", par Odile Foucaud, in L'Auta, n 548, p. 195-209.

- 18/10/1989 : « La bataille de Saint-Sernin ». La Dépêche du Midi / Philippe Emery.

- 19/10/1989 : « Manufacture-Saint-Sernin : même combat ! ». La Dépêche du Midi. 
- 20/10/1989 : « Plaidoyer pour garder Saint-Sernin ». La Dépêche du midi.

- 23/10/1989 : « Pétition pour Saint-Sernin », La Dépêche du Midi.

- 26/10/1989 : «Le conseil général apporte sa pierre ». La Dépêche du Midi / G. S.

- 26/10/1989 : « Non à la dérestauration ». La Dépêche du Midi.

- 28/10/1989 : «Saint-Sernin : arrêtez tout ! ». La Dépêche du Midi / Philippe Emery.

- 31/10/1989 : «Saint-Sernin : le mépris des compétences locales ». La Dépêche du Midi.

- 31/10/1989 : «Arnaud-Bertrand satisfait ». La Dépêche du Midi.

- 31/10/1989 : «A chacun sa pétition ». La Dépêche du Midi.

- 02/11/1989 : «Contre la dérestauration ». La Dépêche du Midi.

- 02/11/1989 : «Les “ sages ” de Llabrès7 ». La Dépêche du Midi.

- 02/11/1989 : « Nos lecteurs ont la parole ». La Dépêche du Midi.

- 03/11/1989 : « La basilique fait le pied de grue ». Le Journal de Toulouse / Christian Maillebieu.

- 14/11/1989 : « Les Amis de la terre et de Saint-Sernin communiquent ». La Dépêche du Midi.

- 15/11/1989 : Des parrains prestigieux au chevet de la basilique ". La Dépêche du Midi / Philippe Emery.

- 16/11/1989 : « Saint-Sernin : le jugement dernier ». La Dépêche du Midi / Philippe Emery.

- 19/11/1989 : «Pour préserver Saint-Sernin »8. Toulouse-Actualités.

- 19/11/1989 : « Saint-Sernin : querelle de clocher ». Sud-Ouest Dimanche / Robert Coustet.

- 20/11/1989 : «Priez pour Saint-Sernin ». Sud-Ouest Dimanche.

- 21/11/1989 : «La Société Archéologique du Midi de la France prend position ». La Dépêche du Midi.

- 21/11/1989 : « Saint-Sernin de Toulouse : bagarre archéologique ». Le Figaro / Yvan Christ.

- 23/11/1989 : « Manne sur les Augustins et Saint-Bertrand-de-Comminges ». La Dépêche du Midi / Dominique Vieu.

- 23/11/1989 : « Saint-Sernin, selon Yves Boiret ». La Dépêche du Midi.

- 23/11/1989 : «Les Toulousains de Toulouse montent au créneau ” / Lettre de l'association.

- 24/11/1989 : « Saint-Sernin : faut-il Viollet-le-Duc?». Le Quotidien de Paris / Bruno Foucart.

- 24/11/1989 : «La restauration selon Boiret ». Le Journal de Toulouse / Christian Maillebiau.

- 24/11/1989 : “Et le beau, dans tout ça ?”. La Dépêche du Midi / Philippe Emery. 
- 24/11/1989 : " Jacques Lévy9 saisit le ministre de la Culture ». La Dépêche du Midi.

- 25/11/1989 : « La guerre des “ mirandes ” de Saint-Sernin ». Le Monde / Philippe Dagen.

- 28/11/1989 : «La “ Dérestauration ” serait confirmée ». La Dépêche du Midi / Philippe Emery.

- 29/11/1989 : «Le projet de restauration de Saint-Sernin accepté ». Le Monde.

- 29/11/1989 : « Saint-Sernin de Toulouse sera restaurée ». La Croix.

- 30/11/1989 : «Le professeur Durliat : “ on va désacraliser Saint-Sernin ” ». La Dépêche du Midi / Philippe Emery.

- 30/11/1989 : "Deux télégrammes “ made in USA ” ". La Dépêche du Midi.

- Novembre 1989 : «Basilique Saint-Sernin : quel visage en l'an 2000 ». ToulouseActualités / Abbé Jean Rocacher.

- 01/12/1989 : « La “ guerre des mirandes ” continue ». Le Monde / Philippe Dagen.

- 01/12/1989 : « Patrice Béghain : “ personne n’a le monopole du sacré ». La Dépêche Du Midi.

- 01/12/1989 : «Recours en grâce du maire de Toulouse ? ». Le Journal de Toulouse / Pierre Rey.

- 02/12/1989 : « Toulouse : Saint-Sernin sera “ dérestaurée ” ». Le Figaro / Yvan Christ.

- 02/12/1989 : « La lettre à Jack Lang : Baudis consacre Saint-Sernin ». La Dépêche du Midi / Philippe Emery.

- 07/12/1989 : « Tempête sous le toit de la basilique Saint-Sernin ». Libération / Louis Destrem.

- 11/12/1989 : «Contre la dérestauration ». La Dépêche du Midi / Philippe Emery.

- 12/12/1989 : « Baudis invite Lang à une visite guidée ». La Dépêche du Midi / Philippe Emery.

- 12/12/1989 : » Campagne : Touche pas à mon Saint-Sernin! ». La Dépêche du Midi.

- 12/12/1989 : « La Flamme Cathare s'enflamme pour la basilique ». La Dépêche du Midi.

- 12/12/1989 : «Qui étaient les constructeurs de Saint-Sernin ? ». La Dépêche du Midi.

- 14/12/1989 : « L’Amérique au secours de la basilique ». La Dépêche du Midi / Philippe Emery.

- 21/12/1989 : « Remettre les pendules à l'heure. Non à la désinformation à SaintSernin ». Le Journal de Toulouse / Christian Maillebiau.

- 21/12/1989 : « Michel Valdiguié écrit au ministre ». La Dépêche du Midi.

- 22/12/1989 : « Jack Lang ne sera pas le Père Noël! ». La Dépêche du Midi.

- 29/12/1989 : « Saint-Sernin : Baudis et Puel chez Lang ». La Dépêche du Midi / Philippe Emery.

- 31/12/1989 : « Saint-Sernin : en faveur de la restauration ». Toulouse-Actualités. 
- Décembre 1989 : « La Basilique Saint-Sernin sera-t-elle transformée en musée ? », par l'association Les Toulousains de Toulouse, in L'Auta, n 551, p. 304-305.

- Décembre 1989 : « Basilique Saint-Sernin : dernière croisade ». Capitole-Infos, p. 16-17. - 05/01/1990 : « Quand D. Baudis rencontre J. Lang. Saint-Sernin, dérestauration ou pas? ». Le Journal de Toulouse / Christian Maillebiau.

- 05/01/1990 : « D. Baudis chez J. Lang 》, La Dépêche du Midi / Philippe Emery.

- 05/01/1990 : « Saint-Sernin : la troisième voie ». La Dépêche du Midi / Philippe Emery.

- 05/01/1990 : »ASPECT : la solution ! ». La Dépêche du Midi.

- 07/01/1990 : «Saint-Sernin : le comité pour la transparence communique ». ToulouseActualités.

- 11/01/1990 : « Une pétition de plus. Les Toulousains de Paris interviennent pour Saint-Sernin ». La Dépêche du Midi / Françoise Caries.

- 11/01/1990 : «La troisième voie ...impasse ou voie de garage ? ». Le Journal De Toulouse / Christian Maillebiau.

- 13/01/1990 : « Toulouse : polémique autour de la basilique Saint-Sernin. Une “ dérestauration " très contestée ». Les Dernières Nouvelles d'Alsace / Philippe Emery.

- 14/01/1990 : » La bataille de Saint-Sernin : un débat passionné ». Toulouse-Actualités / Abbé Jean Rocacher.

- 17/01/1990 : «La Société Archéologique divisée ». La Dépêche du Midi.

- 22/01/1990 : « Saint-Sernin : le roman d'une querelle ». Le Point / Jean Pierrard.

- 31/01/1990 : « Saint-Sernin : tout un roman! ». Le Point / Agnès Cazenave.

- 05/02/1990 : «Bicentenaire du département : une Révolution de retard ». La Dépêche du Midi / Bertrand Durand.

- 28/02/1990 : «L'Affaire de Saint-Sernin de Toulouse ; “ Restaurateurs ” et

“dérestaurateurs " ». Le Figaro / Léon Pressouyre.

- 03/03/1990 : « Prière pour Saint-Sernin ». Le Monde / Michel Parent.

- 18/03/1990 : «Basilique Saint-Sernin. Mise au point ». Toulouse-Actualités..

- 25/03/1990 : «Les Amis de Saint-Sernin témoignent de leur vitalité ». ToulouseActualités.

- 13/04/1990 : « Jack Lang devrait bientôt parler ». La Dépêche du Midi / F. C.

- Avril 1990 : «Au sujet de Saint-Sernin », par l'association Les Toulousains de Toulouse, in L'Auta, $\mathrm{n}^{\circ}$ 555, p. 122-126.

- Avril 1990 : « Restauration. Chefs-d'œuvre en valeur ». Capitole-infos, p. 14-17.

- 27/05/1990 : «Visite incognito : Jack Lang à Saint-Sernin ». La Dépêche du Midi / Philippe Emery.

- 07/06/1990 : « Saint-Sernin, comme en 1860 ». La Dépêche Du Midi / F.C.

- 08/06/1990 : «Querelle de clocher. Flash-Back pour Saint-Sernin ». Le Journal de Toulouse / Christian Maillebiau. 
- 08/06/1990 : «Querelle autour d'un clocher ». La Dépêche du Midi / Philippe Emery.

- 08/06/1990 : « Toulouse. Lang approuve la dérestauration de Saint-Sernin ». La Croix.

- 08/06/1990 : « Saint-Sernin : la guerre des mirandes est finie ». Le Monde / Frédéric Edelmann.

- 15/06/1990 : « Les anti-Boiret campent sur leurs positions ». La Dépêche du Midi / Philippe Emery.

- 15/06/1990 : «Des associations toulousaines “squattent" les jardins de la basilique ; Sauvons Saint-Sernin! ». Le Journal de Toulouse / Christian Maillebiau.

- 16/06/1990 : « Non à la mutilation de Saint-Sernin ! ». L'Humanité.

- 16/06/1990 : « Saint-Sernin, un millier de signatures après un jour d'occupation ». La Dépêche du Midi / Philippe Emery.

- 17/06/1990 : « Saint-Sernin, Montségur, même combat. Et si les débats s'ouvraient? ». Le Journal de Toulouse / Christian Maillebiau.

- 17/06/1990 : «A l'assaut de la dérestauration ». La Dépêche du Midi / Philippe Emery.

- 21/06/1990 : « Peser le pour et le contre ». Le Journal de Toulouse / Christian Maillebiau.

- 21/06/1990 : » Lettre du Conseil Régional de l'Ordre des Architectes ». Le Journal de Toulouse.

- 23/06/1990 : «Bernard Durand à Patrice Béghain : “ nous ne sommes pas des hooligans! " ». La Dépêche du Midi.

- 24/06/1990 : «J. Lang condamne Viollet-le-Duc ». Toulouse-Actualités.

- 26/06/1990 : «L'occupation de la basilique se poursuit ». La Dépêche du Midi / Philippe Emery.

- 26/06/1990 : « La lutte finale ? ». Le Journal de Toulouse / Christian Maillebiau.

- 27/06/1990 : «Les plumes à l'assaut : une surélévation inutile et néfaste ». La Dépêche du Midi.

- 28/06/1990 : «Lang dit non à Baudis ». La Dépêche du Midi / Philippe Emery.

- 28/06/1990 : « Nos lecteurs ont la parole ». La Dépêche du Midi.

- 28/06/1990 : «Un référendum local ». Le Journal de Toulouse / Christian Maillebiau.

- 30/06/1990 : « La bataille de Saint-Sernin continue ... ». La Dépêche du Midi / Philippe Emery.

- 30/06/1990 : «Courriers des lecteurs ». La Dépêche du Midi.

- 01/07/1990 : « Les anti-Boiret lèvent le camp ». Le Journal de Toulouse / Christian Maillebiau.

- 01/07/1990 : « Saint-Sernin : lettre du maire de Toulouse au Président de la République ». Toulouse-Actualités.

- 07/07/1990 : « Portet, Saint-Sernin, même combat ». La Dépêche du Midi / Philippe Emery.

- 07/07/1990 : » Saint-Sernin : 12850 lettres au Président ». La Dépêche du Midi. 
- 09/07/1990 : «Querelles autour du clocher de Saint-Sernin ». Libération /Christian Durand.

- 16/07/1990 : « Saint-Sernin : les architectes avec Boiret ». La Dépêche du Midi.

- 17/07/1990 : « Robert Loïdi : “Jack Lang n'est pas un barbare ” ». La Dépêche du Midi.

- 22/07/1990 : « Basilique Saint-Sernin : le point de vue des architectes ». ToulouseActualités.

- 24/07/1990 : » Reprise des travaux à Saint-Sernin ; le roman continu ». Le Journal de Toulouse.

- 25/07/1990 : » Cessez-le-feu ». La Dépêche du midi.

- 25/07/1990 : « Nos lecteurs ont la parole ». La Dépêche du Midi.

- 29/07/1990 : « Manifeste pour la conservation de Saint-Sernin ». Toulouse-Actualités.

- 31/07/1990 : « Saint-Sernin, coup de Jarnac ». La Dépêche du Midi.

- 31/07/1990 : « 14000 signatures, ça prouve quoi ?». La Dépêche du Midi.

- Juillet 1990 : » Basilique Saint-Sernin, transformée par décret ». Capitole-Infos.

- Juillet 1990 : « L'affaire Saint-Sernin » Marcel Cornu.

- 23/08/1990 : « De Cracovie à Auschwitz, seules les pierres témoignent » Le Monde / Frédéric Edelmann.

- 24/08/1990 : « Le roman d'une épopée ». L'Opinion Indépendante du Sud-Ouest / Christian Saint-Laurent ${ }^{10}$.

- 04/09/1990 : » Au “ chevet" d'une malade ». Le Journal de Toulouse / Christian Maillebiau.

- 13/09/1990 : « Saint-Sernin : des Toulousains reçus prochainement à l'Elysée. Le rendez-vous de l'impossible ». Le Journal de Toulouse / Christian Maillebiau.

- 10/11/1990 : « French architecture. The way it was ». The Economist.

- 16/11/1990 : «Saint-Sernin : restauration chic ou toc ?». La Dépêche du Midi / Philippe Emery.

- Novembre 1990 : » Saint-Sernin : fin d'un chapitre », par Jean Deilhes, in L'Auta, n 561, p. 291-317.

- Mars 1991 : «Saint-Sernin : parenthèse refermée ». Connaissance des arts / Yves Boiret. - 15/02/1991 : « Formules ...lapidaires ». Le Journal de Toulouse / Christian Maillebiau.

- Février 1991 : « Sous la restauration ...la Restauration! ». La Dépêche du Midi / Isabelle Deluze.

- 21/04/1991 : « Dominique Letellier, le passionné ». La Dépêche du Midi / Jean-Jacques Rouch.

- Juin 1991 : « Viollet-le-Duc a perdu une bataille ». Connaissance des arts / Bruno Foucart.

- Décembre 1991 : «La “ dérestauration” de Saint-Sernin de Toulouse ». Encyclopedia Universalis, p. 460-461 / Marcel Durliat. 
- 12/08/1993 : « Saint-Sernin “ dé-violletisée ” 》. L'Express / Anne Pons.

\section{NOTES}

1. . Bruno Foucart, «L'exposition Viollet-le-Duc au Grand Palais à Paris (1979-1980), Viollet-le-Duc à Pierrefonds et dans l'Oise, Paris, Éditions du patrimoine, 2008, p. 2 et suiv. ("Idées et débats »).

2. . Bruno Foucart, «Viollet-le-Duc et la restauration », Pierre Nora (dir.), Les Lieux de mémoire, tome II, « La Nation », Paris, Gallimard (collection « Quarto »), 1997, p. 1617.

3. . Ibid., 1616.

4. 4. Marcel Durliat, "La Restauration de Saint-Sernin de Toulouse. Aspects doctrinaux", Monuments historiques, $\mathrm{n}^{\circ} 112,1980$, p. 103.

5. . Marie Soula, Saint-Sernin de Toulouse. Les enjeux d'une restauration, 1815-1880, mémoire de maîtrise, sous la dir. de Alain Corbin, Paris, Panthéon-Sorbonne, UFR d'histoire, 1995, p. 96.

6. . Cette citation est empruntée à Louis Barron, La Garonne, Paris, Éd. Enri Laurens, s. d. (entre 1863 et 1873), selon le mémoire de Marie Soula, op. cit.., p. 35.

7. . Bérénice Waty, L'Affaire des mirandes à la basilique de Saint-Sernin de Toulouse, Toulouse, DRAC Midi-Pyrénées, 2000, 70 p.

8. . Titre de l'article dans Le Monde, 25 novembre 1989.

1. . Bruno Foucart, « Viollet-le-Duc et la restauration », op. cit., p. 1633.

2. . Yves Boiret, « Problèmes de la restauration », Monuments historiques nº 112, 1980, p. 54-59.

3. . Jean Rochacher, Mosé Biago Moliterni, Saint-Sernin de Toulouse, basilique romane, Toulouse, Éditions Privat, 1993, p. 69.

4. . Trois signataires à ce courrier: Michèle Pradalier-Schlumberger, Louis Peyrusse et Jean Rocacher. Nous nous sommes entretenue avec ce dernier à deux reprises en avril 2000.

5. . Odile Foucaud était doctorante à l'époque. Elle a soutenu une thèse en histoire à Toulouse en 1989, intitulée Jacques-Jean Esquié (1817-1884), architecte à Toulouse. Concernant le document inédit qu'elle a trouvé et étudié, on peut indiquer un de ses articles, « La restauration de Saint-Sernin de Toulouse de 1860 à 1862. 29 nouveaux documents iconographiques signés de Viollet-le-Duc et Esquié », Bulletin monumental n 4, vol. 147, 1989, p. 333-344.

6. . Dans une note relative à ce dossier, on lit cette phrase symptomatique: "Les Toulousains vont recommencer à gronder!». La Rue de Valois aurait donc cherché l'apaisement (fonds d'archives de Jack Lang, ministre de la culture. Institut des mémoires de l'édition contemporaine. Consultation le 18 nov. 2008).

7. . Le 27 octobre 1989, une motion municipale suspendant le commencement et le financement des travaux est adoptée à l'unanimité, annulant les clivages politiques locaux. À sa suite, le conseil général adopte un texte similaire.

8. . Entretien réalisé le 17 mai 2000.

9. . La Dépêche du Midi, 24 nov. 1989.

10. . Du fait de l'usage du parvis de la Basilique pour les "puces », institution toulousaine du dimanche.

11. . La Dépêche du Midi, 12 déc. 1989.

12. . Rocacher, Pradalier, le père Jugla en charge de la basilique et Odile Foucaud. Nous rencontrerons le père Jugla le 10 avril 2000.

13. . La Dépêche du Midi, 30 nov. 1989.

14. Entretien réalisé le 19 mai 2000.

15. . « Plus de quatre-vingt kilos », se souvient Durand.

16. . La Dépêche du Midi, 15 juin 1990.

17. Entretien réalisé en avril 2000. 
18. . Le Journal de Toulouse, 21 juin 1990.

19. . Après vingt articles et lettres ouvertes de ses opposants, Yves Boiret prend enfin la parole dans un article de La Dépêche du Midi le 23 nov. 1989.

20. . La Dépêche du Midi, 13 janv. 1990.

21. . "L'affaire des peintures murales ", « le projet des vitraux » et « l'affaire des cryptes » : trois épisodes de tensions fortes qui se crispent sur le sort de la Basilique. Voir Marie Soula, op. cit..

22. . Michel Marié, "Questions introductives pour un séminaire de réflexion ", Dossiers des séminaires Technique, territoire et société (8-9), MELTM, DRI, 1989, p. 27.

23. . Guy Di Méo, "Productions des identités et attachement au lieu», Yvon Lamy (dir.), L'Alchimie du patrimoine; discours et politiques, Bordeaux, la Maison des sciences de l'homme d'Aquitaine, 1996, p. 265-266.

1. . Henri Pradalier, «Saint-Sernin médiéval », Saint-Sernin de Toulouse, trésors et métamorphoses, Toulouse, Musée Saint-Raymond, 1989, p. 25.

2. . André Micoud, "Musée et patrimoine: deux types de rapport aux choses et au temps", Hermès, $\mathrm{n}^{\circ} 20,1996$, p. 117.

3. . Cas de la manufacture des tabacs, du tracé des lignes de métro, par exemple.

4. . Yvon Lamy, "Le Creuset du patrimoine collectif ", L'Alchimie du patrimoine; discours et politiques, op. cit., p. 19.

5. . NIMBY : « Not In My BackYard».

1. . Bruno Foucart, op. cit., 1997, p. 1631.

2. . Jean-Michel Leniaud, Chroniques patrimoniales, Paris, Éditions Norma, 2001, p. 476.

3. Ibid.

4. 40. Daniel Fabre, «Conclusion de la journée du 7 janvier », Jacques Le Goff (dir.), Patrimoine et passions identitaires, Paris, Fayard, 1998, p. 285.

5. . Ibid.

6. 1. À l'époque, directeur des services de la DRAC, à Toulouse.

7. 2. Conseiller municipal de Toulouse

8. 3. Présentation du comité international de parrainage ; lettre ouverte.

9. 4. Président du groupe socialiste au conseil municipal de Toulouse, à l'époque.

10. Christian Saint-Laurent s'avère être Christian Maillebiau, du Journal de Toulouse.

\section{RÉSUMÉS}

Peut-on restaurer la basilique de Toulouse sans créer de polémique ? Non a priori. Viollet-le-Duc au XIX ${ }^{\mathrm{e}}$ siècle avait subi les foudres des élites locales. Yves Boiret en 1989 connaîtra pareille querelle. Notre article veut relater cette controverse à travers l'énonciation des faits qui l'ont rythmée. Au-delà du conflit architectural, s'est jouée une crise qui a vu la population et les associations prendre position pour le respect de l'intégrité de leur monument, bien métonymique de la cité palladienne. La polémique en elle-même et sa médiatisation seront explicitées, ainsi que son rôle dans l'affirmation d'une identité toulousaine mise à mal dans les années 1980

Can the basilica of Toulouse be restored without causing controversy? At first sight, the answer is no. During the 19th century, Viollet-le-Duc had experienced the wrath of the local elite. In 1989, Yves Boiret will undergo such a quarrel. Our article wants to relate this debate by reporting the facts which punctuated it. Beyond the architectural conflict, a crisis blew up which saw the 
population and the associations taking a stand for the integrity of their monument to be respected, a monument very metonymic of the palladian city. The argument itself and its mediatization will be clarified, as well as its role in the assertion of a Toulousian identity badly damaged in the 1980 s.

Ist es möglich, die Basilika von Toulouse zu restaurieren, ohne dabei eine Polemik auszulösen? Anscheinend nicht. Im 19. Jahrhundert zog sich Viollet-le-Duc den Zorn der lokalen Eliten zu. Yves Boiret erfuhr im Jahre 1989 die gleiche Aufregung. Der vorliegende Artikel zeichnet die Kontroverse anhand ihrer Darstellungsweise nach. Jenseits des Konflikts um die Architektur entstand eine Debatte, in der sich die Bevölkerung und Vereinigungen für die Achtung der Integrität des palladianistischen Monuments eingesetzt haben. Die Polemik sowie ihre Vermittlung wird erläutert und in ihrer Bedeutung für die mitunter krisenhafte Toulouser Identität der $80^{\mathrm{er}}$ Jahre aufgezeigt.

\section{AUTEUR}

\section{BÉRÉNICE WATY}

Bérénice Waty est diplômée de l'institut d'études politiques de Rennes et docteure en anthropologie culturelle de l'École des hautes études en sciences sociales de Paris, en 2006. Son doctorat a porté sur les pratiques de lecture et les relations à l'objet-livre chez les grands lecteurs (personnes lisant 25 livres et plus par an, selon la catégorie du ministère de la culture). Sa thèse, dirigée par Daniel Fabre, s'intitule : L'île lettrée. Voyage au pays des grands lecteurs. Enquête ethnologique d'un groupe statistique de lecteurs. Chercheuse au Laboratoire d'anthropologie et d'histoire de l'institution de la culture (IIAC - LAHIC - UMR 8177), elle poursuit ses recherches autour de la lecture (pratiques et discours des enfants de 3-6 ans ; les bloggeurs de livres). En parallèle, depuis 2000, elle travaille sur les phénomènes de contestation patrimoniale, menée par des associations citoyennes. Elle se penche également sur l'arrivée sur le Net de ces mouvements qui relatent ainsi leurs actions. Courriel : berenice.waty@voila.fr. 